TS-DET 93-034

June 9, 1993

\title{
Stability of Philips XP2012 and XP2008 PMT's
}

\begin{abstract}
S. Delchamps
Results are presented for a 96-hour stability test of three (3) XP2012 PMTs and one (1) XP2008 PMT. These 1.5" diameter, 10-stage PMT's were used in the UA2 experiment and are candidates for some parts of the CDF plug upgrade calorimeter. The four PMT's tested appear to be just as stable as the Hamamatsu R4125's reported on in an earlier memo (TS-DET 93-024.)
\end{abstract}

Three (3) Philips XP-2012's and one (1) Philips XP2008 were held at high voltages corresponding to gains of $2.5 \times 10^{\wedge} 5$. (We ran these PMT"s at the nominal gain corresponding to the hadronic calorimeter, because it seems more likely at this time that if they were used, they would be used in that part of the detector.)

The PMT's were exposed at fifteen minute intervals to 500 flashes (flash rate $\sim 10 \mathrm{~Hz}$ ) of light emerging from a $10 \mathrm{~cm}$ long $1 \mathrm{~mm}$ Bicron BCF91 A wavelength shifter fiber coupled to a $\sim 1 \mathrm{~m}$ long clear fiber. The flashes were produced with the laser-based PMT test system at Lab 2 [1].

Raw data files are in the area

\section{FNALV::PRJ\$ROOT:[TS_CDF_PRJ.STABILITY.ST052893]}

and the PAW output files, plots, and numerical data reported here can be reproduced in the manner described in Note 2.

A single Hamamatsu R580-17 PMT (serial number WA2216) was used to monitor the laser light intensity on a flash-by-flash basis. Figure 1a shows the average response of this PMT, normalized to its initial value, as a function of time. Changes in this qunatity reflect changes in the average laser output with time, as well as changes in the average monitor PMT intrinsic response. Figure 1b shows the "normalized corrected response" or NCR [see Note 3 for an explanation of this important quantity] of a PIN diode, run at -90 $\mathrm{V}$ bias and exposed to light fromt the same system as the WA2216 and other PMT"s, as described in the reference in Note 1.

Figures $1 \mathrm{c}$ and $1 \mathrm{~d}$ show the temperatures given by two thermal probes placed at different locations in the test chamber. The increase in temperature at the very end of the run corresponds to occupation of the laboratory by a number of people after the three-day weekend during which most of the test run took place.

Table 1 shows the type, serial numbers, high voltages, and stability results of the Philips PMT's participating in the test.

Distribution: W. Koska, J. Strait 
Results: Figures $2 a-2 d$ show the NCR's for the four Philips PMT's under test. Figures $2 a-2 c$ correspond to the three XP2012's, and Figure $2 d$ shows the result for the single XP2008 in the test. Several features are apparent in these plots:

(1) First, the "up-turn" in NCR shown by all PMT's at the end of the run does not match any corresponding behavior in the monitor tube (Figure 1a), and is not understood at this time. The upturn in the NCR's begins well before the increase in temperature in Figures 1c and 1d, and in any case we would expect a decrease in the NCR for a temperature increase in the test chamber.

(2) The scatter in the NCR's is large compared to the typical scatters shown by previously tested PMTs. This may be seen by comparing Figures $4 a / 4 b, 5 a / 5 b$, and $6 \mathrm{a} / 6 \mathrm{~b}$ which show the NCR's of three Hamamatsu R4125 PMT's run at the same high voltages in the present test ("b" figures) and in an earlier test ("a" figures.) The dispersions of the NCR's in the present test are clearly worse. The NCR scatters seem highly correlated between the four PMTs under test. It has now been determined that recabling of the system just before the run reported on here led to a x10 increase in the monitor PMT noise.

The NCR of PMT 12003 was used to "renormalize" the other three Philips PMTs. Figures 3a, 3b, and 3d show the NCR's for the other two XP2012's and the single XP2008 divided by the NCR of the PMT shown in Figure 3c, in order to remove the common fluctuations due to the monitor PMT problem. The three renormalized PMT"s now show much less dispersion in their NCR's. (12003 of course is the same as in Figure 1c, since it was used to "renormalize" the other three PMT's.) The NCR of 8002 shows a steady upward drift. A linear fit gives a drift of $0.44 \pm .01 \% /$ day.

The second to last column of Table 1 gives the maximum range (in percent of the mean) of the NCR for the 24-hour period in which this parameter was maximum, for times between 20 hours and 80 hours The early times are left off as part of the assumed "runin" period of the PMT"s, and the times beyond 80 hours are left off because they include the unexplained "up-turn." The last column gives the maximum NCR ranges for the three PMT's for which "renormalized" NCR's were obtained. Here, times greater than 20 hours are taken into account.

The maximum NCR ranges in Table 1 may be compared with the values for Hamamatsu R4125's reported earlier in TS-DET 93-024. The mean and sigma of the most recent batch of Hamamatsu R4125's are shown here in the last row of Table 1. The Philips PMT's appear to be just as stable as the Hamamatsu R4125's. 


\begin{tabular}{|l|l|l|l|l|}
\hline Tube Type & Serial Number & $\begin{array}{l}\text { High Voltage } \\
\text { (Volts) } \\
(\text { Gain }= \\
\left.2.5 \times 10^{\wedge} 5\right)\end{array}$ & $\begin{array}{l}\text { Maximum NCR } \\
\text { Range } \\
\text { (\% of mean } \\
\text { over 24 hours) } \\
\text { [uncorrected] }\end{array}$ & $\begin{array}{l}\text { Maximum NCR } \\
\text { Range } \\
\text { (\% of mean } \\
\text { over 24 hours) } \\
\text { [corrected] }\end{array}$ \\
\hline XP 2012 & 12001 & 1365 & 3.23 & 1.25 \\
\hline & 12002 & 1351 & 3.16 & 0.65 \\
\hline & 12003 & 1314 & 2.85 & - \\
\hline XP 2008 & & & & 0.84 \\
\hline & & 1195 & 2.88 & \\
\hline R4125 & & & & $\begin{array}{c}1.52 \pm 0.28 \\
\text { (average over } \\
8 \text { PMTs in } \\
\text { TS-DET 93-024) }\end{array}$ \\
\hline
\end{tabular}

Table 1. Stability Results for Philips 1 1/2" PMT's 


\section{Notes}

1. S. Delchamps, and B. Kinney. "Preliminary PMT Stability Measurements with Laser Light Source." TS-DET 93-002.

2. The NCR or "normalized corrected response" is the "corrected response" (CR) divided by its initial value. The CR can be defined for a PMT as

$$
\mathrm{CR}=\left\{\sum\left(\mathrm{PMT}_{\mathrm{i}} / \mathrm{CORR}_{\mathrm{i}}\right)\right\} / 500
$$

where $\mathrm{PMT}_{\mathrm{i}}$ is the pedestal-subtracted response of the PMT for the $i$ th flash, and CORR is the response of the correction device (in the case of the test reported in this memo, the monitor PMT WA2216), for the ith flash. The correction device in principle removes the variations in the PMT response which are due only to variations in the laser light level. It is assumed that the correction device is perfectly stable, and that the ratio

$$
\mathrm{R}=\text { Light delivered to the correction device/ Light delivered to the PMT }
$$

for each laser flash is a constant. (See TS-DET 93-002 for more details.)

3. To reproduce the plots in this memo, first go to the project area

$$
\text { FNALV::PRJ\$ROOT:[TS_DET_PRJ.STABILITY.ST052893] }
$$

You must have privileges to use this area. Contact Steve Delchamps (FNALV::DELCHTS) to find out about this. Now, execute the command procedure LASER2.COM. Then, execute the command procedure RENORM.COM. In PAW, you may now use the file LASER2.KUMAC to produce the plots shown in this memo. To obtain the stability numbers shown in the last columns of Table 1 , you must run

[STABILITY]STABLE2 on the ".sum2" files, and

[STABILITY]STABLE on the ".sum3" files. The first program leaves out times after 80 hours, and the second includes them. 

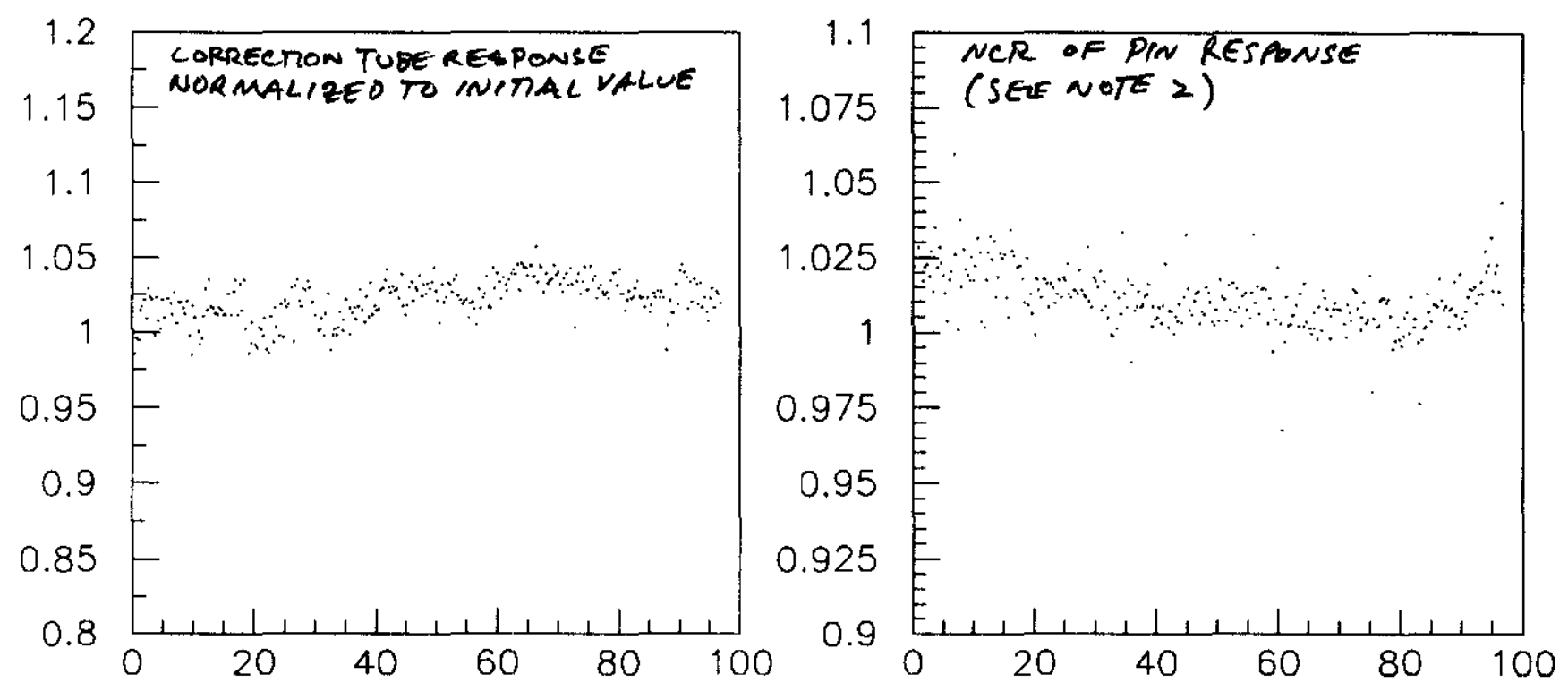

Fig. 1a WA2216 vs Time (hrs)

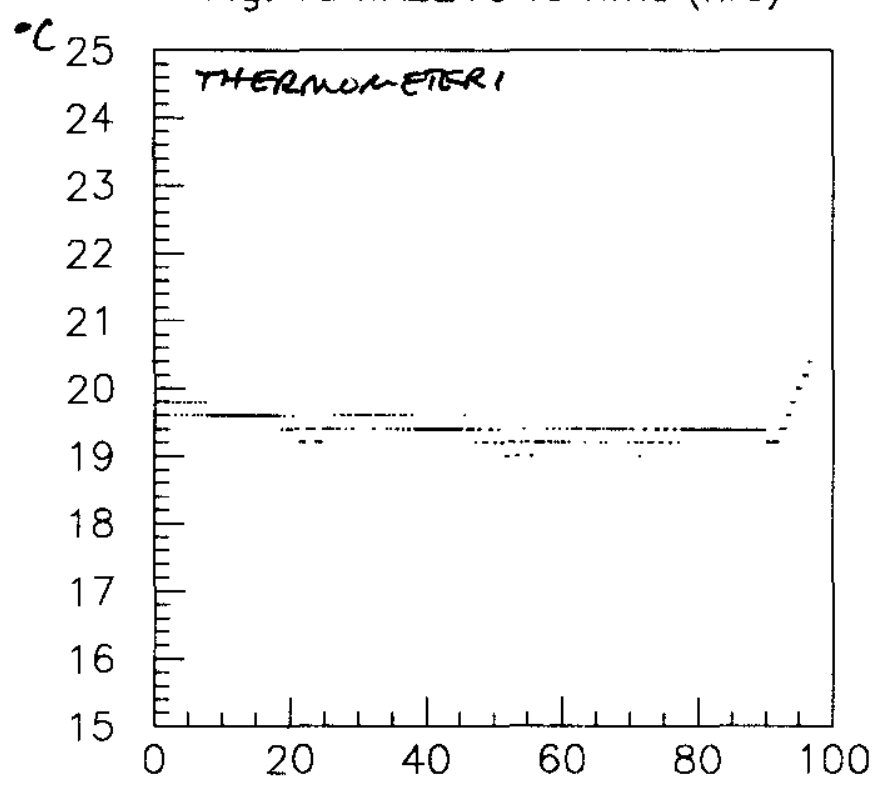

Fig. 1b PIN 2 vs Time (hrs)

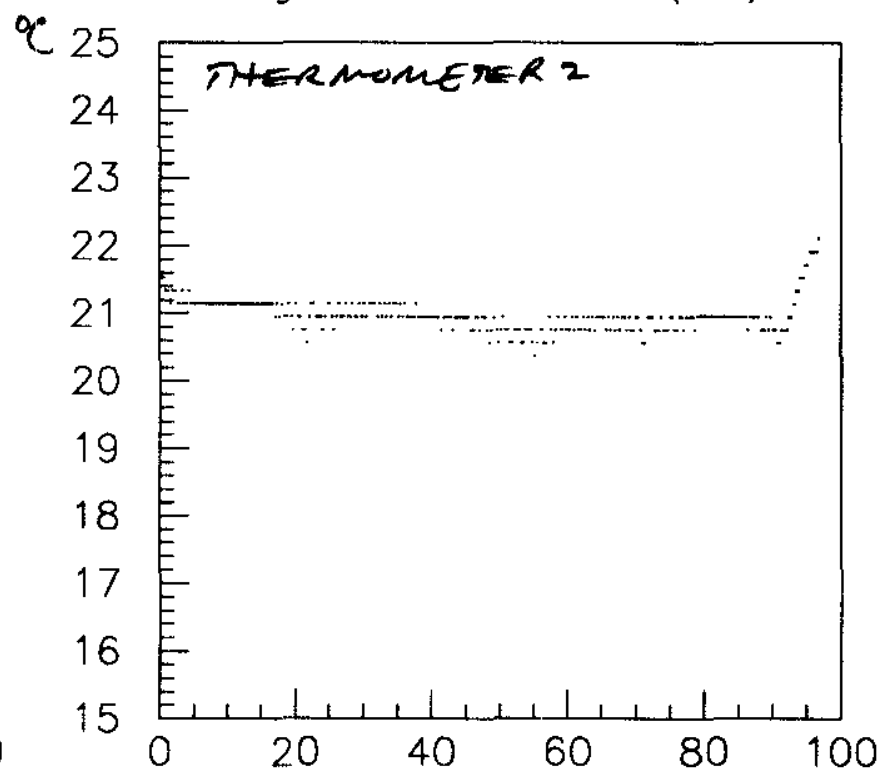

Fig. 1c TEMPA vs Time (hrs)

Fig. 1d TEMPB vs Time (hrs) 


\section{NCR (SEE NOTE 2) FOR PHLIPS I/2" PMTS}

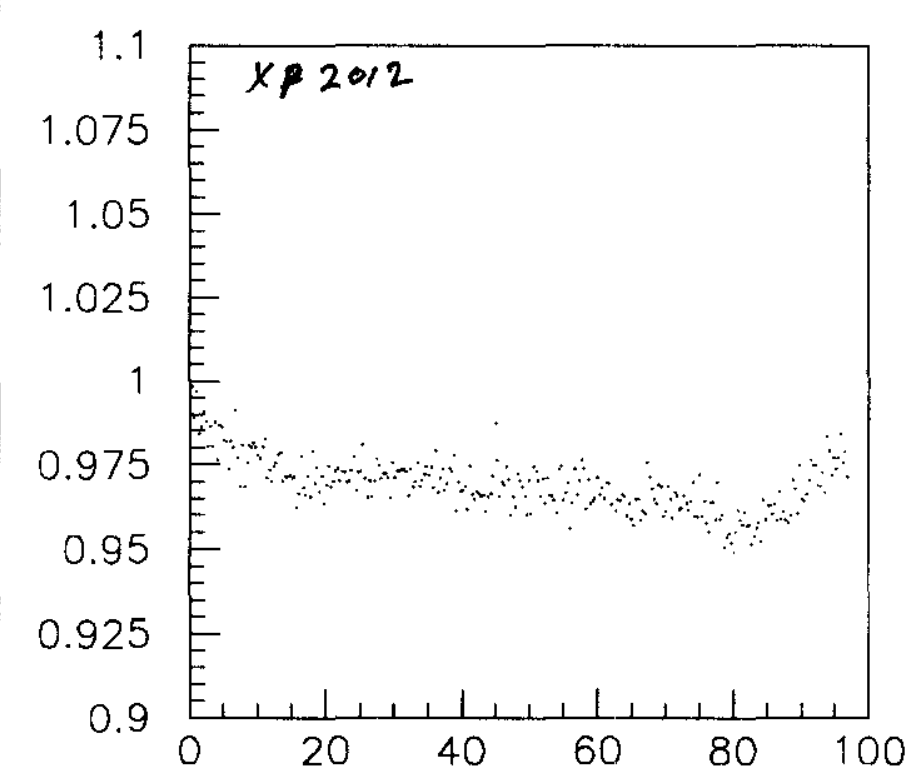

Fig. 2a 12001 vs Time (hrs)

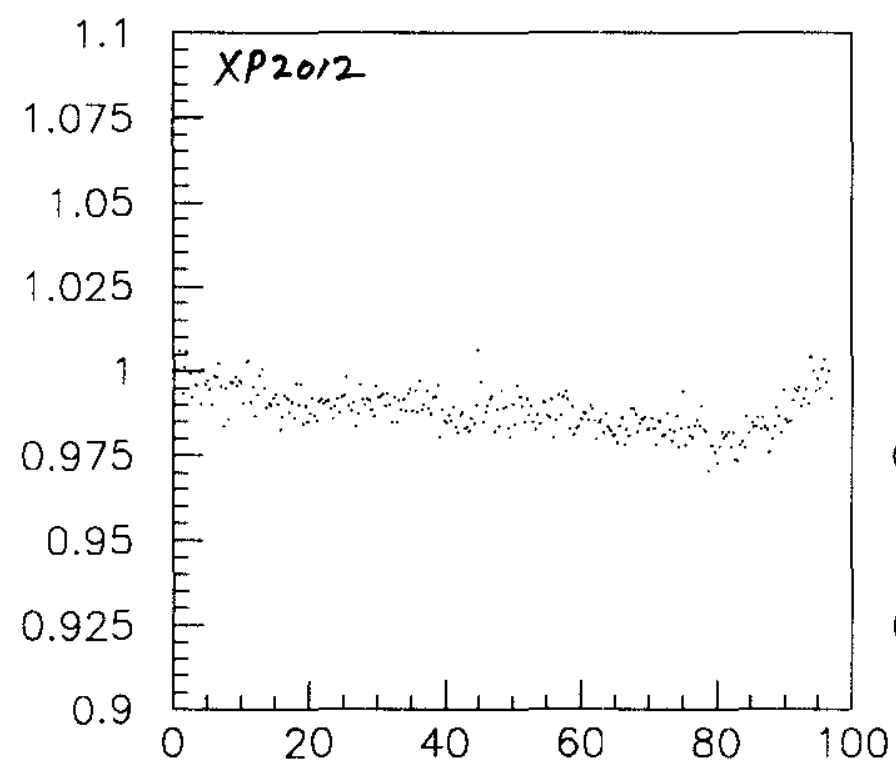

Fig. 2c 12003 vs Time (hrs)

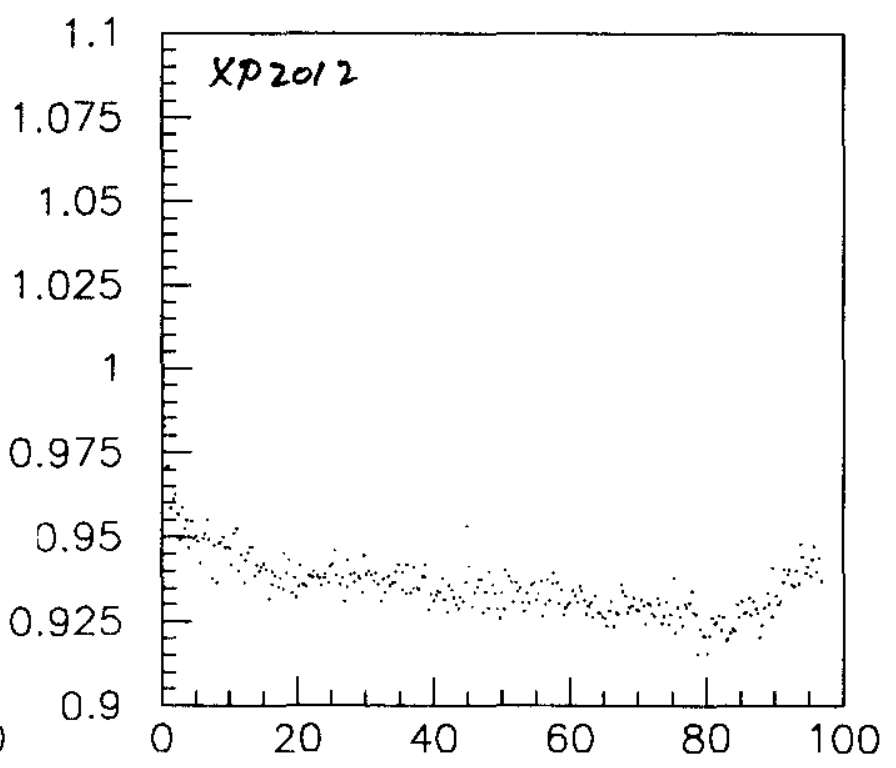

Fig. 2b 12002 vs Time (hrs)

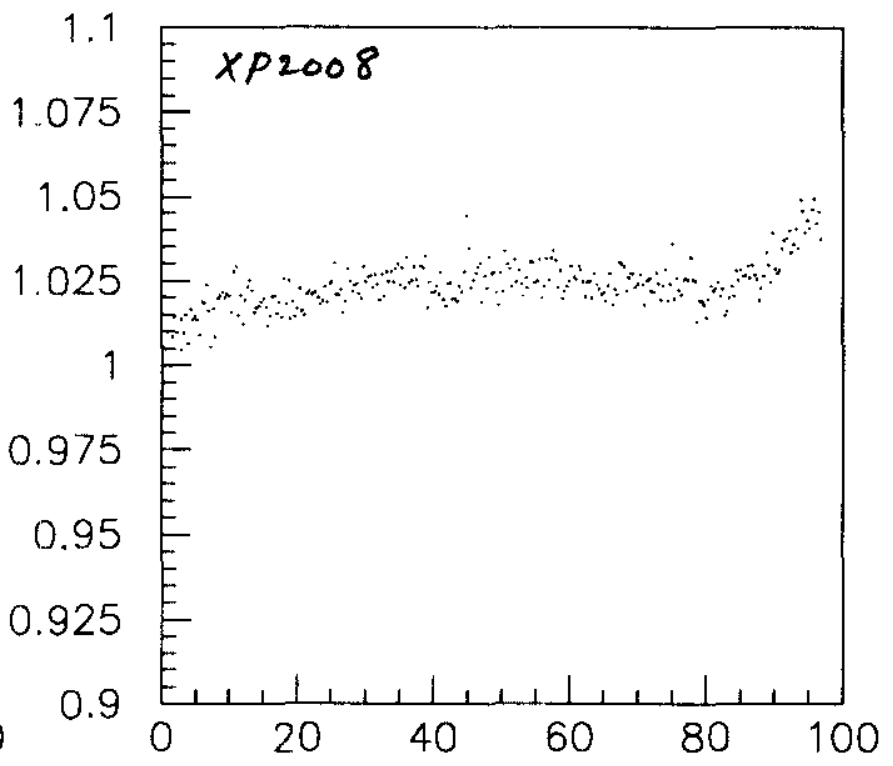

Fig. 2d 8002 vs Time (hrs) 


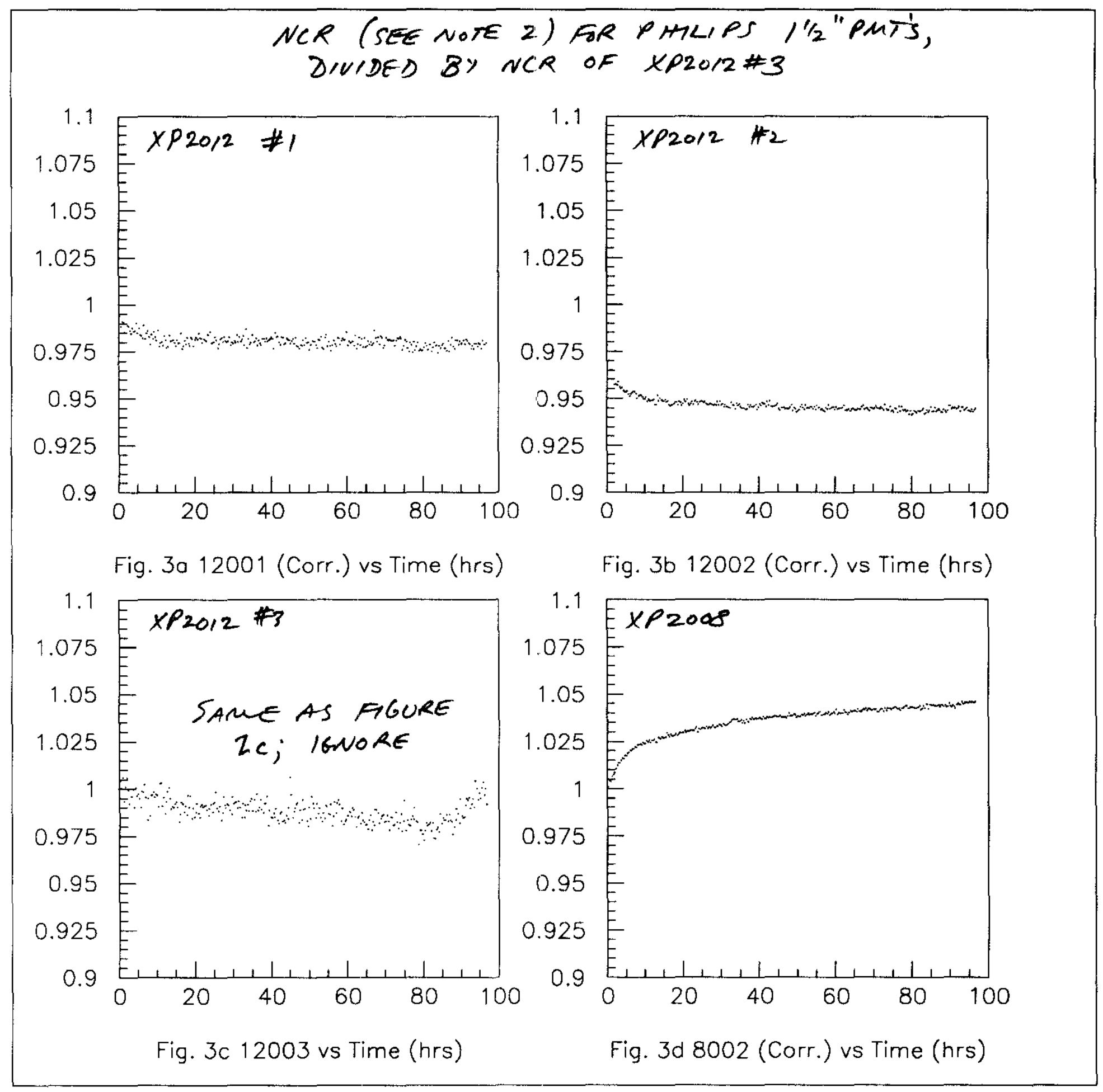




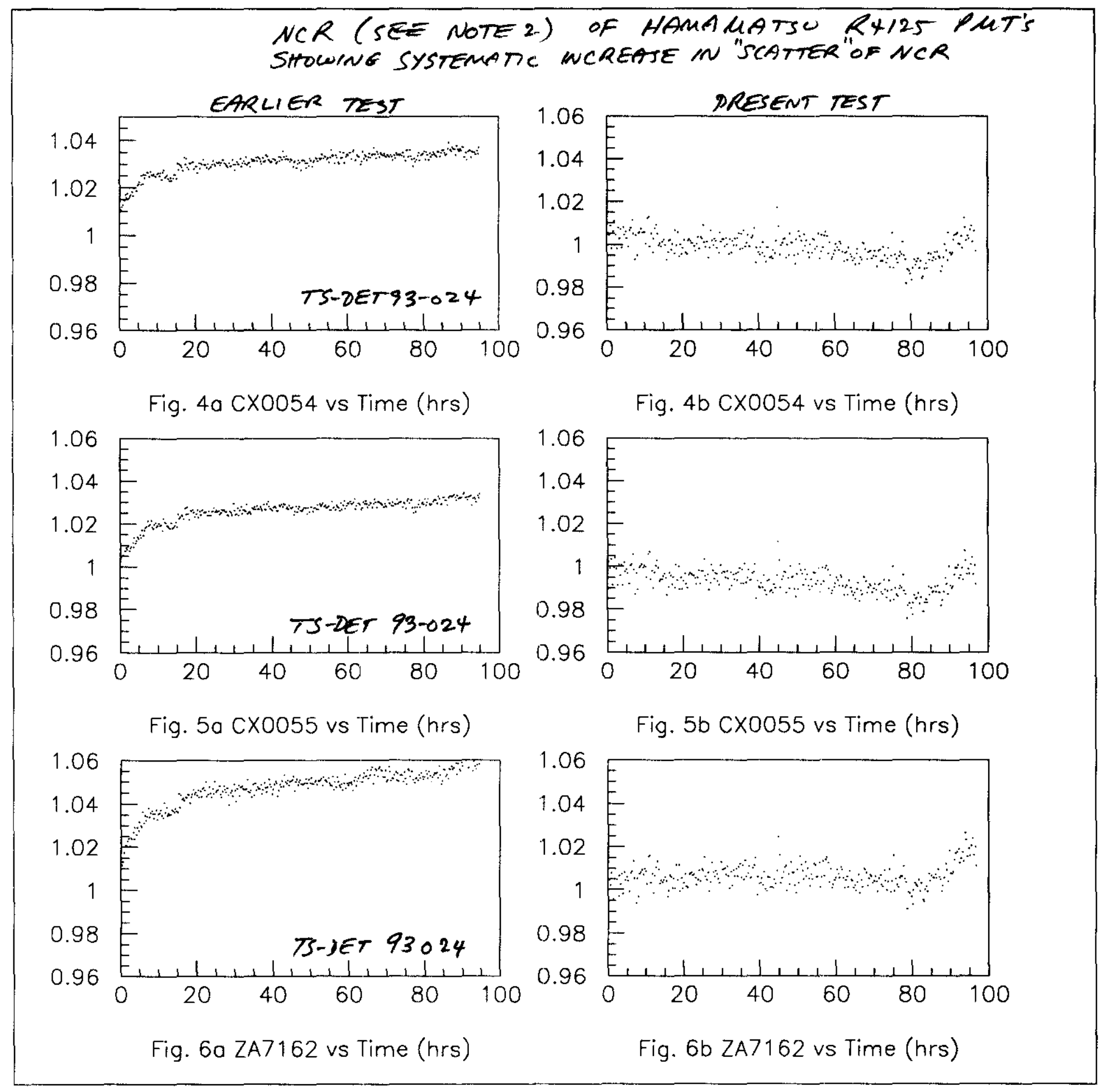

